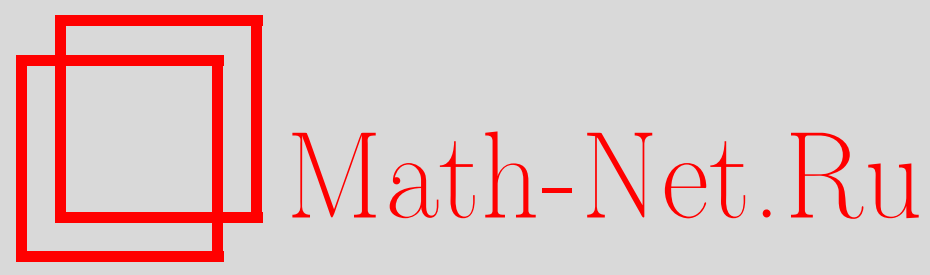

Р. А. Заторский, Определители треугольных матриц и траектории на диаграммах Ферре, Матем. заметки, 2002, том 72, выпуск $6,834-852$

DOI: https://doi.org/10.4213/mzm471

Использование Общероссийского математического портала Math-Net.Ru подразумевает, что вы прочитали и согласны с пользовательским соглашением http://www . mathnet.ru/rus/agreement

Параметры загрузки:

IP : 3.89 .185 .249

26 апреля 2023 г., 12:49:15

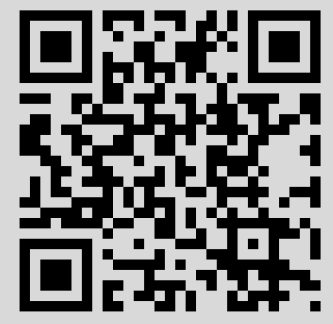




\section{ОПРЕДЕЛИТЕЛИ ТРЕУГОЛЬНЫХ МАТРИЦ И ТРАЕКТОРИИ НА ДИАГРАММАХ ФЕРРЕ}

\section{Р. А. Заторский}

Рассматриваются некоторые функции, заданные на множестве целых треугольных матриц, а также их модификации. Эти функции являются аналогами классических функций определителя и перманента и дают мощный аппарат исследования последовательностей, порожденных линейньми рекуррентными уравнениями. При помощи модификаций этих функций решается задача о траекториях на диаграммах Ферре и устанавливаются новые комбинаторные тождества.

Библиограбия: 4 названия.

1. Треугольные матрицы, параопределители и параперманенты. В этом пункте мы дадим определения параопределителя и параперманента. Более основательно эти функции треугольных действительных матриц изучаются в [1].

ОпРЕДЕЛЕнИЕ 1.1. Треугольную таблицу чисел вида

$$
A=\left(\begin{array}{cccc}
a_{11} & & & \\
a_{21} & a_{22} & & \\
\ldots \ldots & \ldots & \ldots & \\
a_{n 1} & a_{n 2} & \ldots & a_{n n}
\end{array}\right)_{n}
$$

назовем треугольной матрицей, а число $n$ - ее порядком.

Каждому элементу $a_{i j}$ матрицы (1.1) поставим в соответствие $(i-j+1)$ элементов $a_{i k}, k=j, \ldots, i$, которые назовем производными әлементами матрицы, порожденньми ключевым әлементом $a_{i j}$. Ключевой элемент одновременно является и своим производным элементом. Произведение всех производных элементов, порожденных ключевым элементом $a_{i j}$, обозначим через $\left\{a_{i j}\right\}$ и назовем факториальным произведением ключевого элемента $a_{i j}$, т.е. $\left\{a_{i j}\right\}=\prod_{k=j}^{i} a_{i k}$.

ОПРЕДЕЛЕНиЕ 1.2. Набор ключевых элементов матрицы (1.1) назовем нормальным набором этой матрицы, если они порождают множество производных элементов мощности $n$, каждые два из которых не лежат в одном столбце этой матрицы.

Пусть $A(n)$ - множество упорядоченных разбиений $\alpha=\left(\alpha_{1}, \ldots, \alpha_{r}\right)$ натурального числа $n$. Известно, что $|A(n)|=2^{n-1}$. Пусть $\alpha=\left(\alpha_{1}, \ldots, \alpha_{r}\right) \in A(n)$, тогда каждой компоненте $\alpha_{s}, s=1, \ldots, r$, этого разбиения поставим в соответствие ключевой элемент $a_{i j}$ матрицы (1.1) при помощи следующего алгоритма:

$1^{\circ}$ начать; 
$2^{\circ} j=1 ; s=0 ; i=0$;

$3^{\circ} s=s+1 ; i=i+\alpha_{s}$ элемент $(s)=a_{i j}$;

$4^{\circ}$ если $s<r$, то $j=j+\alpha_{s}$; перейти к $3^{\circ}$;

$5^{\circ}$ конец.

Каждому нормальному набору $а$ ключевых элементов припишем знак $(-1)^{\varepsilon(a)}$, где $\varepsilon(a)$ - сумма всех индексов ключевых элементов этого набора.

ОПРЕДЕЛЕНИЕ 1.3. Параопределителем треугольной матрицы (1.1) назовем число

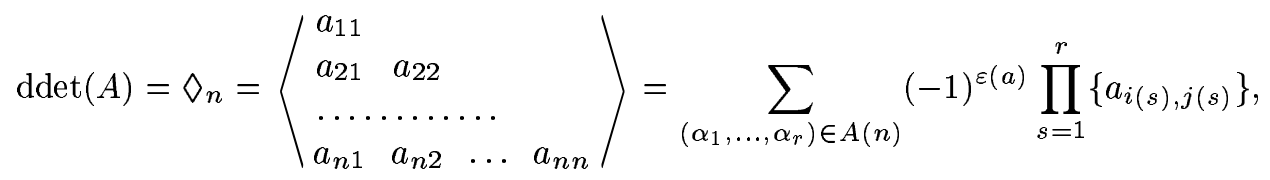

где $a_{i(s), j(s)}$ - ключевой элемент, соответствующий $s$-й компоненте разбиения $\alpha=$ $\left(\alpha_{1}, \ldots, \alpha_{r}\right)$, a $(-1)^{\varepsilon(a)}$ - знак нормального набора $a$ ключевых элементов.

Аналогично понятию параопределителя введем понятие параперманента.

ОПРЕДЕЛЕНИЕ 1.4. Параперманентом матрицы (1.1) назовем число

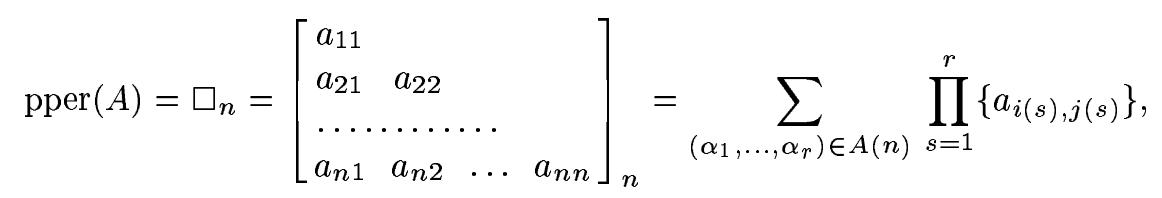

где $a_{i(s), j(s)}$ - ключевой элемент, которьй соответствует $s$-й компоненте разбиения $\alpha=\left(\alpha_{1}, \ldots, \alpha_{r}\right)$ из множества $A(n)$.

Следует отметить, что параопределитель и параперманент матрищы (1.1) состоят всего из $2^{n-1}$ слагаемых. Поэтому, если выразить члены некоторой последовательности $\left\{u_{n}\right\}_{n=1}^{\infty}$ в виде параопределителя или параперманента, достигается значительная экономия числа операций. Поскольку для каждой числовой последовательности, порожденной линейньм рекуррентным уравнением с некоторьми начальными условиями, можно построить соответствующий параперманент $\square_{n}$ (см. теорему 2.1), то понятия параопределителя и параперманента во многих случаях становятся незаменимьми.

Введем для параопределителя и параперманента понятия, аналогичные классическим понятиям минора и алгебраического дополнения. С этой целью каждому элементу $a_{i j}$ матрицы (1.1) поставим в соответствие треугольную таблицу элементов этой матрицы с этим элементом в левом нижнем углу, которую назовем углом матрииы и обозначим через $R_{i j}$. Углу $R_{i j}$ принадлежат только те элементы $a_{r s}$ матрицы (1.1), индексы которых удовлетворяют соотношениям $j \leqslant s \leqslant r \leqslant i$. Очевидно, что угол $R_{i j}$ является матрищей $(i-j+1)$-го порядка. В дальнейшем будем считать, что

$$
\operatorname{ddet}\left(R_{01}\right)=\operatorname{ddet}\left(R_{n, n+1}\right)=\operatorname{pper}\left(R_{01}\right)=\operatorname{pper}\left(R_{n, n+1}\right)=1 .
$$

Если в угле $R_{i j} j$-й столбец заменить соответствующими элементами $k$-го столбца $(k<j)$, а все остальные элементы угла оставить неизменньми, то такой угол обозначим через $R_{i ; j \mapsto k}$. Будем считать, что $\operatorname{ddet}\left(R_{n ; n+1 \mapsto n}\right)=\operatorname{pper}\left(R_{n ; n+1 \mapsto n}\right)=0$. 
ОПРЕДЕЛЕНИЕ 1.5. Прямоугольную таблицу элементов матрицы (1.1) назовем впuсанной в эту матрицу, если одна ее вершина совпадает с элементом $a_{n 1}$, а противоположная к ней вершина - с элементом $a_{i i}, i=1, \ldots, n$. Обозначим эту таблицу через $T(i)$.

ПРИМЕЧАНИЕ 1.1. Если в определении $1.5 i=1$ или $i=n$, то вписанная прямоугольная таблища вырождается соответственно в первый столбец или последнюю строчку.

ОПРЕДЕЛЕНИЕ 1.6. Алгебраическими дополнениями $D_{i j}, P_{i j}$ к факториальному произведению ключевого элемента $a_{i j}$ матрицы (1.1) назовем соответственно числа

$$
D_{i j}=(-1)^{i+j} \operatorname{ddet}\left(R_{j-1,1}\right) \operatorname{ddet}\left(R_{n, i+1}\right), \quad P_{i j}=\operatorname{pper}\left(R_{j-1,1}\right) \operatorname{pper}\left(R_{n, i+1}\right),
$$

где $R_{j-1,1}$ и $R_{n, i+1}$ - углы матрицы (1.1).

Интересным является тот факт, что некоторые свойства параопределителей аналогичны свойствам классических определителей (см. [1]).

2. Применение параперманентов к решению линейных рекуррентных уравнений. Приведем две важные теоремы, которые позволяют решить линейное рекуррентное уравнение $k$-го порядка с постоянными коэффициентами, избегая решения соответствующего алгебраического уравнения того же порядка. Доказательства приведенных ниже теорем можно найти в [1].

Пусть задано линейное рекуррентное уравнение $k$-го порядка

$$
u_{n+k}=a_{1} u_{n+k-1}+\cdots+a_{k} u_{n}, \quad 1 \leqslant k \leqslant n, \quad n \in \mathbb{N},
$$

с начальньми условиями

$$
u_{i}=b_{i}, \quad i=1, \ldots, k, \quad b_{i} \in \mathbb{R}
$$

ТЕОРема 2.1. Параперманент вида

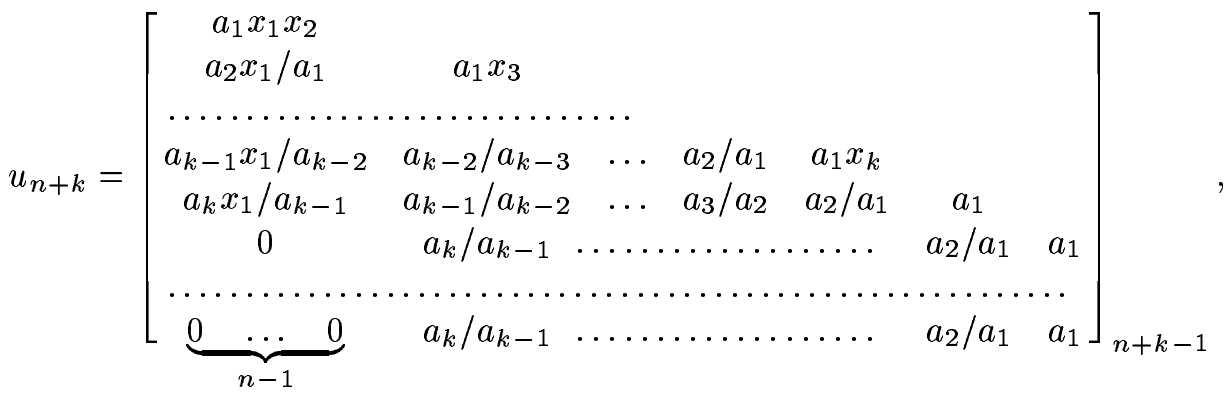

$$
\begin{aligned}
& u_{1}=x_{1} \text {, }
\end{aligned}
$$

әде поправки $x_{i}$ определяются из равенств

$$
x_{1}=b_{1}, \quad x_{i}=\frac{b_{i}}{a_{1} b_{i-1}+a_{2} b_{i-2}+\cdots+a_{i-1} b_{1}}, \quad i=2, \ldots, k,
$$

является решением уравнения (2.1) с начальными условиями (2.2), причем $b_{r}=0$, если $r \leqslant 0$. 
Примечание 2.1. Решение уравнения (2.1) с начальньми условиями (2.2) при помощи теоремы о связи параопределителя и параперманента (см. [1]) можно выразить через параопределитель.

Для рекуррентного уравнения (2.1) начальные условия вида

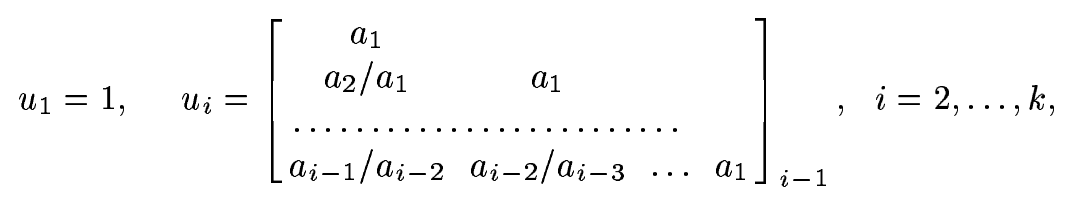

назовем стандартными начальными условиями.

ТЕОрема 2.2. Линейное рекуррентное уравнение $k$-го порядка $(2.1)$ с постоянными коэффициентами и стандартными начальными условиями (2.3) имеет решение вида

$$
\begin{aligned}
& u_{1}=1
\end{aligned}
$$

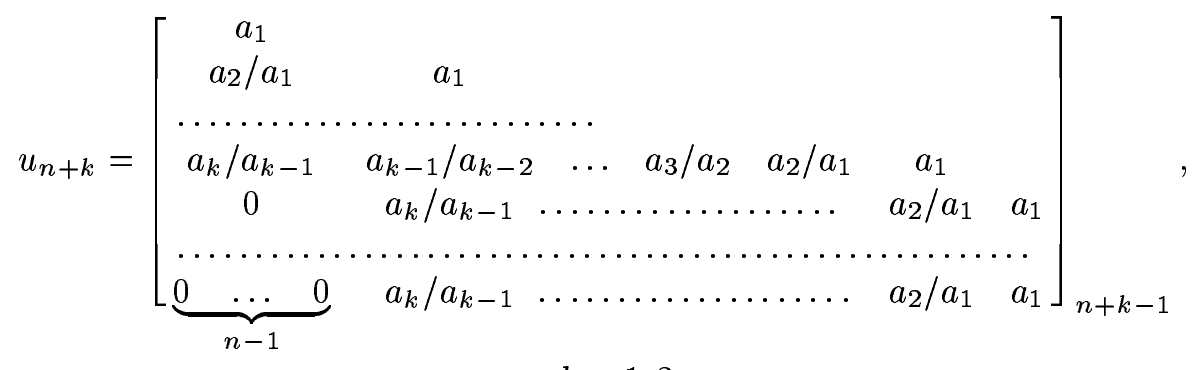

$$
\begin{aligned}
& n, k=1,2, \ldots \text {. }
\end{aligned}
$$

3. Модификации параопределителей и параперманентов. В этом пункте мы введем некоторые модификации параопределителей и параперманентов, которые назовем $F$-параопределителями и $F$-параперманентами.

Пусть задана матрища (1.1). Каждому элементу $a_{i j}, 1 \leqslant j \leqslant i \leqslant n$, этой матрицы поставим в соответствие $i-j+1$ элементов $w_{i k}, k=j, \ldots, i$, которые назовем виртуальны.ми әлементами этой матрицы, порожденными ключевым элементом $a_{i j}$. Ключевой элемент одновременно является и своим виртуальным элементом. Виртуальные элементы играют роль вспомогательных элементов и предназначены облегчить доказательство некоторых утверждений. Если виртуальные элементы задаются посредством одного из равенств $w_{i k}=a_{i j}+k-j$ или $w_{i k}=a_{i j}-k+j, k=j, \ldots, i$, то следующие два выражения:

$$
\begin{aligned}
& \left\{a_{i j}\right\}^{\circ}=\frac{a_{i j}^{\overline{i-j+1}}}{(i-j+1) !}=\frac{a_{i j}\left(a_{i j}+1\right) \cdots\left(a_{i j}+i-j\right)}{(i-j+1) !} \\
& \left\{a_{i j}\right\}_{\circ}=\frac{a_{i j}^{i-j+1}}{(i-j+1) !}=\frac{a_{i j}\left(a_{i j}-1\right) \cdots\left(a_{i j}-i+j\right)}{(i-j+1) !}
\end{aligned}
$$

назовем соответственно верхним и нижним факториальными произведениями ключевого элемента $a_{i j}$. В равенствах (3.1) и (3.2) мы пользуемся следующими обозначениями возрастающих и убьвающих факториальных степеней:

$$
x^{\bar{m}}=x(x+1) \cdots(x+m-1), \quad x \underline{m}=x(x-1) \cdots(x-m+1)
$$

(см. $[2$, с. 67]). 
Таким образом, производные элементы ключевого элемента заменяются соответственньми виртуальными элементами, а набор ключевых элементов считается нормальным, если он порождает множество виртуальных элементов мошности $n$, каждые два из которых не принадлежат одновременно одному столбцу этой матрицы.

ОПРЕДЕЛЕНИЕ 3.1. Верхним (нижним) F-параопределителем матрицы (1.1) назовем соответственно числа

$$
\begin{aligned}
& \operatorname{ddet}^{\circ}(A)=\diamond_{n}^{\circ}=\sum_{\left(\alpha_{1}, \ldots, \alpha_{r}\right) \in A(n)}(-1)^{\varepsilon(a)} \prod_{s=1}^{r}\left\{a_{i_{s}, j_{s}}\right\}^{\circ} \\
& \operatorname{ddet}_{\circ}(A)=\diamond_{n^{\circ}}=\sum_{\left(\alpha_{1}, \ldots, \alpha_{r}\right) \in A(n)}(-1)^{\varepsilon(a)} \prod_{s=1}^{r}\left\{a_{i_{s}, j_{s}}\right\}_{\circ},
\end{aligned}
$$

где $a_{i_{s}, j_{s}}-$ ключевой элемент, соответствующий $s$-й компоненте разбиения $\alpha ;\left\{a_{i_{s}, j_{s}}\right\}^{\circ}$, $\left\{a_{i_{s}, j_{s}}\right\}_{\circ}-$ соответственно верхнее и нижнее факториальные произведения ключевого элемента $a_{i_{s}, j_{s}}$, которые задаются соответственно равенствами $(3.1),(3.2)$, a $(-1)^{\varepsilon(a)}-$ множитель, определяющий знак нормального набора $a$ ключевых элементов.

Аналогично определению $F$-параопределителя введем понятие верхнего и нижнего $F$-параперманента.

ОПРЕДЕЛЕНИЕ 3.2. Верхним (нижним) F-параперманентом матрицы (1.1) назовем соответственно числа

$$
\begin{aligned}
& \operatorname{pper}^{\circ}(A)=\square_{n}^{\circ}=\sum_{\left(\alpha_{1}, \ldots, \alpha_{r}\right) \in A(n)} \prod_{s=1}^{r}\left\{a_{i_{s}, j_{s}}\right\}^{\circ}, \\
& \operatorname{pper}_{\circ}(A)=\square_{\circ n}=\sum_{\left(\alpha_{1}, \ldots, \alpha_{r}\right) \in A(n)} \prod_{s=1}^{r}\left\{a_{i_{s}, j_{s}}\right\}_{\circ},
\end{aligned}
$$

где $a_{i_{s}, j_{s}}-$ ключевой элемент, соответствующий $s$-й компоненте разбиения $\alpha, \mathrm{a}\left\{a_{i_{s}, j_{s}}\right\}^{\circ}$, $\left\{a_{i_{s}, j_{s}}\right\}_{\circ}-$ соответственно верхнее и нижнее факториальные произведения этого ключевого элемента.

ОПРедЕЛЕНИЕ 3.3. Алгебраическими дополнениями $D_{i j}^{\circ}, D_{i j \circ}, P_{i j}^{\circ}, P_{i j \circ}$ к верхнему и нижнему факториальным произведениям ключевого элемента $a_{i j}$ назовем соответственно числа

$$
\begin{gathered}
D_{i j}^{\circ}=(-1)^{i+j} \operatorname{ddet}^{\circ}\left(R_{j-1,1}\right) \cdot \operatorname{ddet}^{\circ}\left(R_{n, i+1}\right), \\
D_{i j \circ}=(-1)^{i+j} \operatorname{ddet}_{\circ}\left(R_{j-1,1}\right) \cdot \operatorname{ddet}_{\circ}\left(R_{n, i+1}\right), \\
P_{i j}^{\circ}=\operatorname{pper}^{\circ}\left(R_{j-1,1}\right) \cdot \operatorname{pper}^{\circ}\left(R_{n, i+1}\right), \quad P_{i j \circ}=\operatorname{pper}_{\circ}\left(R_{j-1,1}\right) \cdot \operatorname{ddet}_{\circ}\left(R_{n, i+1}\right) .
\end{gathered}
$$

4. Свойства $F$-параопределителей треугольных матриц. Для $F$-параопределителей справедливы утверждения и следствия, аналогичные соответственным утверждениям и следствиям для параопределителей (см. [1]). Приведем их без доказательства. 
УТВеРЖДЕнИЕ 4.1 (разложение по элементам вписанной таблицы). Пусть $A-$ матрича (1.1), а $T(i)$ - некоторая вписанная в нее прямоугольная таблича әлементов. Тогда выполняются следуюшие равенства:

$$
\begin{aligned}
\operatorname{ddet}^{\circ}(A) & =\sum_{s=1}^{i} \sum_{r=i}^{n}\left\{a_{r s}\right\}^{\circ} D_{r s}^{\circ} \\
\operatorname{ddet}_{\circ}(A) & =\sum_{s=1}^{i} \sum_{r=i}^{n}\left\{a_{r s}\right\}_{\circ} D_{r s \circ}
\end{aligned}
$$

СлЕдСтвиЕ (разложение $F$-параопределителя по элементам первого столбца). $E c л u$ $i=1$, то равенства (4.1), (4.2) примут соответственно вид

$$
\begin{aligned}
& \operatorname{ddet}^{\circ}(A)=\sum_{r=1}^{n}\left\{a_{r 1}\right\}^{\circ} D_{r 1}^{\circ}=\sum_{r=1}^{n}(-1)^{r+1}\left\{a_{r 1}\right\}^{\circ} \operatorname{ddet}^{\circ}\left(R_{n, r+1}\right), \\
& \operatorname{ddet}_{\circ}(A)=\sum_{r=1}^{n}\left\{a_{r 1}\right\}_{\circ} D_{r 1 \circ}=\sum_{r=1}^{n}(-1)^{r+1}\left\{a_{r 1}\right\}_{\circ} t \operatorname{ddet}_{\circ}\left(R_{n, r+1}\right)
\end{aligned}
$$

Справедливо также аналогичное разложение по әлементам последней строчки.

УтвеРждЕниЕ 4.2 (разложение по элементам $j$-го столбца). Для матрищы (1.1) выполняются равенства

$$
\begin{gathered}
\sum_{r=j}^{n}\left\{a_{r j}\right\}^{\circ} D_{r j}^{\circ}=\operatorname{ddet}^{\circ}\left(R_{j-1,1}\right) \cdot \operatorname{ddet}^{\circ}\left(R_{n j}\right), \\
\sum_{r=j}^{n}\left\{a_{r j}\right\}_{\circ} D_{r j \circ}=\operatorname{ddet}_{\circ}\left(R_{j-1,1}\right) \cdot \operatorname{ddet}_{\circ}\left(R_{n j}\right) .
\end{gathered}
$$

УТВеРЖДЕНИЕ 4.3. F-параопределитель блочно-диагональной матрицы (см. [1]), равен произведению F-параопределителей ее блоков.

Приведем несколько соотношений с возрастающими и убывающими факториальными степенями:

$$
\begin{gathered}
\left.\frac{i^{\bar{r}}=\frac{(i+r-1)^{\underline{r}}}{r !}, \quad i \in \mathbb{N}, \quad r \in \mathbb{N}_{0} ;}{r !}, \quad \begin{array}{l}
i \\
r
\end{array}\right)=\frac{i \underline{\underline{r}}}{r !}=\frac{(i-r+1)^{\bar{r}}}{r !}, \quad r \leqslant i, \quad r \in \mathbb{N}_{0}, \quad i \in \mathbb{N}_{0} .
\end{gathered}
$$


УТВЕРЖДЕНИЕ 4.4. Справедливы следующие тождества:

$$
\begin{gathered}
\frac{(r+1)^{\bar{i}}}{i !}=\frac{(i+1)^{\bar{r}}}{r !}, \quad i, r \in \mathbb{N}_{0} ; \\
\frac{r \underline{\underline{i}}}{i !}=\frac{r \frac{r-i}{(r-i) !}, \quad i \leqslant r, \quad i \in \mathbb{N}_{0} ;}{\frac{i^{\bar{r}}}{r !}=\sum_{j=1}^{i} \frac{j^{\overline{r-1}}}{(r-1) !}, \quad r, i \in \mathbb{N} ;} \\
\frac{i \underline{\underline{r}}}{r !}=\sum_{j=r-1}^{i-1} \frac{j \frac{r-1}{(r-1) !},}{(r-1)} \quad r \in \mathbb{N}, \quad r \leqslant i .
\end{gathered}
$$

ДокАЗАТЕЛЬСТво. Докажем тождество (4.7). При $r=1$ имеем

$$
\frac{2 \cdot 3 \cdots i \cdot(i+1)}{1 \cdot 2 \cdots(i-1) \cdot i}=\frac{(i+1)^{\overline{1}}}{1 !}
$$

Пусть тождество (4.7) вьполнено при $r=s$. Докажем его истинность при $r=s+1$. Имеем

$$
\frac{(r+1)^{\overline{s+1}}}{(s+1) !}=\frac{(r+1)^{s}(r+s+1)}{s !(s+1)}=\frac{(s+1)^{\bar{r}}}{r !} \cdot \frac{r+s+1}{s+1}=\frac{(s+2)^{\bar{r}}}{r !} .
$$

Докажем справедливость тождества (4.8). Имеем

$$
\frac{r \underline{i}}{i !} \stackrel{(4.6)}{=} \frac{(r-i+1)^{\bar{i}}}{i !} \stackrel{(4.7)}{=} \frac{(i+1)^{\overline{r-i}}}{(r-i) !} \stackrel{(4.5)}{=} \frac{r \frac{r-i}{(r-i) !}}{} .
$$

Докажем тождество (4.9). Прежде всего докажем, что производящей функцией последовательности $(i+1)^{\bar{r}} / r$ ! является функция $f(x)=(1-x)^{-(r+1)}$. Для этого найдем коэффициент при $x^{i}$ в ее разложении в ряд Маклорена. Имеем

$$
\frac{f^{(i)}(0)}{i !}=\frac{(r+1)^{\bar{i}}}{i !} \stackrel{(4.7)}{=} \frac{(i+1)^{\bar{r}}}{r !}
$$

Таким образом,

$$
(1-x)^{-(r+1)}=\frac{1^{\bar{r}}}{r !}+\frac{2^{\bar{r}}}{r !} x+\cdots+\frac{(i+1)^{\bar{r}}}{r !} x^{i}+\cdots
$$

поэтому коэффищиент при $x^{i-1}$ в произведении $(1-x)^{-1}(1-x)^{-r}$, очевидно, равен

$$
\frac{1^{\overline{r-1}}}{(r-1) !}+\frac{2^{\overline{r-1}}}{(r-1) !}+\cdots+\frac{i^{\overline{r-1}}}{(r-1) !}=\sum_{j=1}^{i} \frac{j^{\overline{r-1}}}{(r-1) !}
$$


Докажем тождество (4.10). Имеем

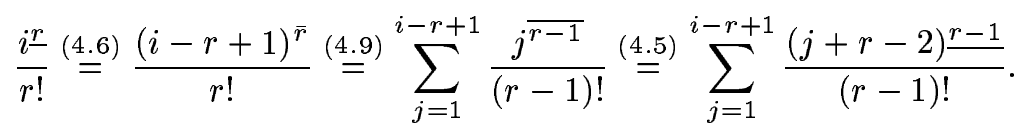

Положив в последней сумме $k=j+r-2$, получим правую часть тождества (4.10).

Докажем несколько утверждений, свойственных только $F$-параопределителям.

Обозначим через

$$
A\left(\begin{array}{l}
j \\
k
\end{array}\right)
$$

матрицу (1.1), в которой к каждому элементу $j$-го столбца прибавлено некоторое целое число $k$, а остальные элементы этой матрицы неизменны.

УтвеРжДЕнИЕ 4.5. Для F-параопределителей выполняются соотношения

$$
\begin{aligned}
\operatorname{ddet}^{\circ}(A) & =\operatorname{ddet}^{\circ}\left(A\left(\begin{array}{c}
j \\
-1
\end{array}\right)\right)+\operatorname{ddet}^{\circ}\left(R_{j-1,1}\right)\left(\operatorname{det}^{\circ}\left(R_{n, j+1}\right)-\operatorname{ddet}^{\circ}\left(R_{n ; j+1 \mapsto j}\right)\right), \\
\operatorname{ddet}_{\circ}(A)= & \operatorname{ddet}_{\circ}\left(A\left(\begin{array}{c}
j \\
-1
\end{array}\right)\right) \\
& \quad+\operatorname{ddet}_{\circ}\left(R_{j-1,1}\right) \cdot\left(\operatorname{ddet}_{\circ}\left(R_{n, j+1}\right)-\operatorname{ddet}_{\circ}\left(R_{n ; j+1 \mapsto j}\left(\begin{array}{c}
j \\
-1
\end{array}\right)\right)\right) .
\end{aligned}
$$

ДокАЗАТЕЛЬСтво. Докажем равенство (4.11) при $j=1$, т.е. докажем равенство

$$
\operatorname{ddet}^{\circ}(A)=\operatorname{ddet}^{\circ}\left(A\left(\begin{array}{c}
1 \\
-1
\end{array}\right)\right)+\operatorname{ddet}^{\circ}\left(R_{n 2}\right)-\operatorname{ddet}^{\circ}\left(R_{n ; 2 \mapsto 1}\right) .
$$

$\mathrm{C}$ этой целью разложим верхний $F$-параопределитель $\operatorname{ddet}^{\circ}(A)$ по элементам первого столбца, используя соотношения (4.3):

$$
\begin{aligned}
\operatorname{ddet}^{\circ}(A) & =\sum_{r=1}^{n}(-1)^{r+1} \frac{a_{r 1}^{\bar{r}}}{r !} \operatorname{ddet}^{\circ}\left(R_{n, r+1}\right) \\
& =\sum_{r=1}^{n}(-1)^{r+1}\left(\frac{\left(a_{r 1}-1\right)^{\bar{r}}}{r !}+\frac{a_{r 1}^{\overline{r-1}}}{(r-1) !}\right) \operatorname{ddet}^{\circ}\left(R_{n, r+1}\right) \\
& =\sum_{r=1}^{n}(-1)^{r+1} \frac{\left(a_{r 1}-1\right)^{\bar{r}}}{r !} \operatorname{ddet}^{\circ}\left(R_{n, r+1}\right)+\sum_{r=1}^{n}(-1)^{r+1} \frac{a_{r 1}^{\bar{r}-1}}{(r-1) !} \operatorname{ddet}^{\circ}\left(R_{n, r+1}\right) \\
& =\operatorname{ddet}^{\circ}\left(A\left(\begin{array}{c}
1 \\
-1
\end{array}\right)\right)+\frac{a_{11}^{\overline{0}}}{0 !} \operatorname{ddet}^{\circ}\left(R_{n 2}\right)+\sum_{r=2}^{n}(-1)^{r+1} \frac{a_{r 1}^{\overline{r-1}}}{(r-1) !} \operatorname{ddet}^{\circ}\left(R_{n, r+1}\right) .
\end{aligned}
$$

Пусть в последней сумме $s=r-1$, тогда

$$
\begin{aligned}
\sum_{r=2}^{n}(-1)^{r+1} \frac{a_{r 1}^{\overline{r-1}}}{(r-1) !} \operatorname{ddet}^{\circ}\left(R_{n, r+1}\right) & =-\sum_{s=1}^{n-1}(-1)^{s+1} \frac{a_{s+1,1}^{\bar{s}}}{s !} \operatorname{ddet}^{\circ}\left(R_{n, s+2}\right) \\
& =\operatorname{ddet}^{\circ}\left(R_{n ; 2 \mapsto 1}\right) .
\end{aligned}
$$


Следовательно, равенство (4.12) истинно.

Разложим верхний $F$-параопределитель

$$
\operatorname{ddet}^{\circ}\left(A\left(\begin{array}{c}
j \\
-1
\end{array}\right)\right)
$$

по элементам вписанной таблищы $T(j)$ :

$$
\operatorname{ddet}^{\circ}\left(A\left(\begin{array}{c}
j \\
-1
\end{array}\right)\right)=\sum_{r=j}^{n} \sum_{s=1}^{j-1} \frac{a_{r s}^{\overline{r-s+1}}}{(r-s+1) !} D_{r s}^{\circ}+\sum_{s=j}^{n} \frac{\left(a_{s j}-1\right)^{\overline{s-j+1}}}{(s-j+1) !} D_{s j}^{\circ}
$$

Вторая сумма равенства (4.13) соответствует разложению этого $F$-параопределителя по элементам $j$-го столбца. Обозначим первую сумму через $\varphi$. Теперь разложим верхний $F$-параопределитель $\operatorname{ddet}^{\circ}(A)$ по элементам таблицы $T(j)$ :

$$
\operatorname{ddet}^{\circ}(A)=\varphi+\sum_{s=j}^{n} \frac{a_{s j}^{\overline{s-j+1}}}{(s-j+1) !} D_{s j}^{\circ} \stackrel{(4.4)}{=} \varphi+\operatorname{ddet}^{\circ}\left(R_{j-1,1}\right) \cdot \operatorname{ddet}^{\circ}\left(R_{n j}\right) .
$$

Представим элементы первого столбца угла $R_{n j}$ в виде $a_{s j}=\left(a_{s j}-1\right)+1, s=j, \ldots, n$, и используем равенство (4.12). Тогда получим

$$
\begin{aligned}
\operatorname{ddet}^{\circ}(A)= & \varphi+\operatorname{ddet}^{\circ}\left(R_{j-1,1}\right) \cdot\left(\operatorname{ddet}^{\circ}\left(R_{n j}\left(\begin{array}{c}
j \\
-1
\end{array}\right)\right)\right. \\
& \left.+\operatorname{ddet}^{\circ}\left(R_{n, j+1}\right)-\operatorname{ddet}^{\circ}\left(R_{n ; j+1 \mapsto j}\right)\right) \\
= & \varphi+\operatorname{ddet}^{\circ}\left(R_{j-1,1}\right) \cdot \operatorname{ddet}^{\circ}\left(R_{n j}\left(\begin{array}{c}
j \\
-1
\end{array}\right)\right) \\
& +\operatorname{ddet}^{\circ}\left(R_{j-1,1}\right) \cdot\left(\operatorname{ddet}^{\circ}\left(R_{n, j+1}\right)-\operatorname{ddet}^{\circ}\left(R_{n ; j+1 \mapsto j}\right)\right) .
\end{aligned}
$$

Поскольку по утверждению 4.2

$$
\operatorname{ddet}^{\circ}\left(R_{j-1,1}\right) \cdot \operatorname{ddet}^{\circ}\left(R_{n j}\left(\begin{array}{c}
j \\
-1
\end{array}\right)\right)=\sum_{s=j}^{n} \frac{\left(a_{s j}-1\right)^{\overline{s-j+1}}}{(s-j+1) !} D_{s j}
$$

то, учитьвая (4.13), получим равенство (4.11).

5. F-параопределители матриц специального вида. Пусть матрица (1.1) задана равенством

$$
a_{i j}=a_{i}-(i-j)+k, \quad 1 \leqslant j \leqslant i \leqslant n .
$$

Обозначим верхний и нижний $F$-параопределители матрицы $(5.1)$, соответственно через $\diamond 1_{n}^{\circ}, \diamond 1_{n \circ}$. 
УТВеРЖДЕНИЕ 5.1. Для верхнего F-параопределителя $\diamond 1_{n}^{\circ}$ выполняется равенство

$$
\diamond 1_{n}^{\circ}=\sum_{j=0}^{n} \frac{(j+1)^{\overline{k-1}}}{(k-1) !} \operatorname{ddet}^{\circ}\left(R_{j 1}\left(\begin{array}{ccc}
1 & \ldots & j \\
-k & \ldots & -k
\end{array}\right)\right),
$$

¿дe $\operatorname{ddet}^{\circ}\left(R_{01}\right)=1, a \operatorname{ddet}^{\circ}\left(R_{n 1}\right)=\diamond 1_{n}^{\circ}$.

ДокАЗАТЕЛЬСтво. Докажем равенство (5.2) при $k=1$. Запишем равенство (4.12) в виде

$$
\operatorname{ddet}^{\circ}(A)-\operatorname{ddet}^{\circ}\left(A\left(\begin{array}{c}
1 \\
-1
\end{array}\right)\right)=\operatorname{ddet}^{\circ}\left(R_{n 2}\right)-\operatorname{ddet}^{\circ}\left(R_{n ; 2 \mapsto 1}\right) .
$$

K правой части равенства (5.3) применимо равенство, аналогичное равенству (5.3), поэтому, продолжая процесс вычислений, получим равенства

$$
\begin{aligned}
& \operatorname{ddet}^{\circ}(A)-\operatorname{ddet}^{\circ}\left(A\left(\begin{array}{c}
1 \\
-1
\end{array}\right)\right)=\operatorname{ddet}^{\circ}\left(R_{n 2}\right)-\operatorname{ddet}^{\circ}\left(R_{n ; 2 \mapsto 1}\right) \\
& \quad=\operatorname{ddet}^{\circ}\left(R_{n 3}\right)-\operatorname{ddet}^{\circ}\left(R_{n ; 3 \mapsto 2}\right)=\cdots=\operatorname{ddet}^{\circ}\left(a_{n}+k\right)-\operatorname{ddet}^{\circ}\left(a_{n}+k-1\right)=1 .
\end{aligned}
$$

Эти равенства вместе с равенством (4.11) дадут равенство

$$
\diamond 1_{n}^{\circ}=\operatorname{ddet}^{\circ}(A)-\operatorname{ddet}^{\circ}\left(A\left(\begin{array}{c}
j \\
-1
\end{array}\right)\right)+\operatorname{ddet}^{\circ}\left(R_{j-1,1}\right) .
$$

При $j=n$ равенство (5.4) имеет вид

$$
\diamond 1_{n}^{\circ}=\operatorname{ddet}^{\circ}(A)=\operatorname{ddet}^{\circ}\left(A\left(\begin{array}{c}
n \\
-1
\end{array}\right)\right)+\operatorname{ddet}^{\circ}\left(R_{n-1,1}\right) .
$$

Применяя к верхнему $F$-параопределителю $\operatorname{det}^{\circ}\left(R_{n-1,1}\right)$ равенство $(5.4)$ при $j=n-1$, получим равенство

$$
\operatorname{ddet}^{\circ}\left(R_{n-1,1}\right)=\operatorname{ddet}^{\circ}\left(R_{n-1,1}\left(\begin{array}{c}
n-1 \\
-1
\end{array}\right)\right)+\operatorname{ddet}^{\circ}\left(R_{n-2,1}\right),
$$

которое вместе с (5.4) даст равенство

$$
\diamond 1_{n}^{\circ}=\operatorname{ddet}^{\circ}\left(R_{n 1}\left(\begin{array}{c}
n \\
-1
\end{array}\right)\right)+\operatorname{ddet}^{\circ}\left(R_{n-1,1}\left(\begin{array}{c}
n-1 \\
-1
\end{array}\right)\right)+\operatorname{ddet}^{\circ}\left(R_{n-2,1}\right) .
$$

Продолжая процесс аналогичньх вычислений, получим равенство

$$
\diamond 1_{n}^{\circ}=\sum_{i=0}^{n} \operatorname{ddet}^{\circ}\left(R_{i 1}\left(\begin{array}{c}
i \\
-1
\end{array}\right)\right)
$$

в котором $\operatorname{ddet}^{\circ}\left(R_{01}\left(\begin{array}{c}0 \\ -1\end{array}\right)\right)=1$. 
Так как в верхнем $F$-параопределителе $\operatorname{ddet}^{\circ}\left(R_{i 1}\left(\begin{array}{c}i \\ -1\end{array}\right)\right)$ соответственные элементы $i$-го и $(i-1)$-го столбцов одинаковые, то, применяя к нему равенство (4.11) при $j=i-1$, получим равенство

$$
\operatorname{ddet}^{\circ}\left(R_{i 1}\left(\begin{array}{c}
i \\
-1
\end{array}\right)\right)=\operatorname{ddet}^{\circ}\left(R_{i 1}\left(\begin{array}{cc}
i-1 & i \\
-1 & -1
\end{array}\right)\right) .
$$

Таким образом, мы приходим к аналогичной ситуации, так как в $F$-параопределителе, лежащем в правой части последнего равенства, соответственные элементы $(i-1)$-го и $(i-2)$-го столбцов равны. Продолжая процесс аналогичных вычислений, мы придем к равенству

$$
\operatorname{ddet}^{\circ}\left(R_{i 1}\left(\begin{array}{c}
i \\
-1
\end{array}\right)\right)=\operatorname{ddet}^{\circ}\left(R_{i 1}\left(\begin{array}{ccc}
1 & \cdots & i \\
-1 & & -1
\end{array}\right)\right),
$$

которое вместе с (5.5) даст равенство

$$
\diamond 1_{n}^{\circ}=\sum_{j=0}^{n} \operatorname{ddet}^{\circ}\left(R_{j 1}\left(\begin{array}{ccc}
1 & \ldots & j \\
-1 & \ldots & -1
\end{array}\right)\right)
$$

т.е. равенство $(5.2)$ при $k=1$. Обозначим $(n-j+1)^{\overline{r-1}} /(r-1)$ ! через $k_{j}(r)$, a

$$
\operatorname{ddet}^{\circ}\left(R_{j 1}\left(\begin{array}{ccc}
1 & \ldots & j \\
-r & \ldots & -r
\end{array}\right)\right)
$$

- через $R_{j}(r)$, тогда равенство (5.2) перепишется в виде

$$
\diamond 1_{n}^{\circ}=\sum_{j=0}^{n} k_{j}(k) R_{j}(k)
$$

Пусть равенство (5.7) выполняется при $k=r$. Докажем его справедливость при $k=r+1$.

Так как $F$-параопределители

$$
\operatorname{ddet}^{\circ}\left(R_{j 1}\left(\begin{array}{ccc}
1 & \ldots & j \\
-r & \ldots & -r
\end{array}\right)\right), \quad j=0, \ldots, n,
$$

являются $F$-параопределителями типа $\diamond 1_{n}^{\circ}$, то к каждому из них применимо равенство (5.6). При этом получим

$$
\operatorname{ddet}^{\circ}\left(R_{j 1}\left(\begin{array}{ccc}
1 & \ldots & j \\
-r & \ldots & -r
\end{array}\right)\right)=\sum_{i=0}^{j} \operatorname{ddet}^{\circ}\left(R_{i 1}\left(\begin{array}{ccc}
1 & \ldots & i \\
-(r+1) & \ldots & -(r+1)
\end{array}\right)\right)
$$

или

$$
\operatorname{ddet}^{\circ}\left(R_{j}(r)\right)=\sum_{i=0}^{j} \operatorname{ddet}^{\circ}\left(R_{i}(r+1)\right)
$$


Подставив (5.8) в (5.7), получим

$$
\diamond 1_{n}^{\circ}=\sum_{j=0}^{n} k_{j}(r) \sum_{i=0}^{j} \operatorname{ddet}^{\circ}\left(R_{i}(r+1)\right)=\sum_{i=0}^{n} \operatorname{ddet}^{\circ}\left(R_{i}(r+1)\right) \cdot \sum_{j=0}^{i} k_{j}(r),
$$

т.е.

$$
k_{i}(r+1)=\sum_{j=1}^{i} k_{j}(r)
$$

Следовательно, для того чтобы доказать справедливость равенства (5.2) при $k=r+1$, необходимо доказать, что коэффициенты

$$
k_{j}(r)=\frac{(n-j+1)^{\overline{r-1}}}{(r-1) !}
$$

удовлетворяют равенству (5.9), которое, очевидно, совпадает с равенством (4.9) из утверждения 4.4. Утверждение 5.1 доказано.

Рассмотрим некоторые простейшие примеры треугольных матриц и их $F$-параопределителей, при помощи которых можно получить двойственные тождества.

Если, например, элементы матрицы (1.1) имеют вид $a_{i j}=a$, то легко доказать равенства

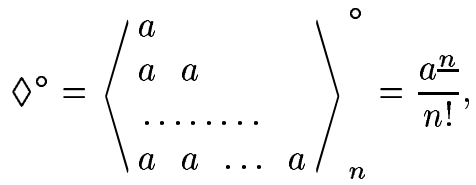

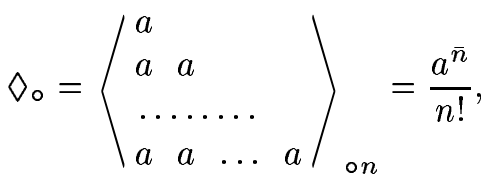

которые являются двойственными равенствами.

После разложения $F$-параопределителей $(5.10),(5.11)$ по элементам $n$-й строки получим соответственно равенства

$$
\begin{aligned}
\diamond_{n}^{\circ} & =\sum_{s=1}^{n}(-1)^{s+n} \frac{a^{\overline{n-s+1}}}{(n-s+1) !} \diamond_{s-1}^{\circ}, \\
\diamond_{n \circ} & =\sum_{s=1}^{n}(-1)^{s+n} \frac{a^{n-s+1}}{(n-s+1) !} \diamond_{s-1 \circ},
\end{aligned}
$$

которые с учетом $(5.10),(5.11)$ дадут соответственно тождества

$$
\begin{aligned}
\frac{a^{\underline{n}}}{n !} & =\sum_{s=1}^{n}(-1)^{s+n} \frac{a^{\overline{n-s+1}}}{(n-s+1) !} \cdot \frac{a^{s-1}}{(s-1) !}=\sum_{i=0}^{n-1}(-1)^{n+i+1} \frac{a^{\overline{n-i}}}{(n-i) !} \cdot \frac{a^{\underline{i}}}{i !} \\
\frac{a^{\bar{n}}}{n !} & =\sum_{s=1}^{n}(-1)^{s+n} \frac{a \frac{n-s+1}{(n-s+1) !}}{(s-1) !}=\sum_{i=0}^{\overline{s-1}}(-1)^{n+i+1} \frac{a \frac{n-i}{(n-i) !}}{\left(s-\frac{a^{i}}{i !}\right.}
\end{aligned}
$$


Если же в тождествах $(5.12),(5.13)$ положить соответственно $a=0,1, \ldots, n-1$ и $a=0,-1, \ldots,-(n-1)$, то они перепишутся в виде

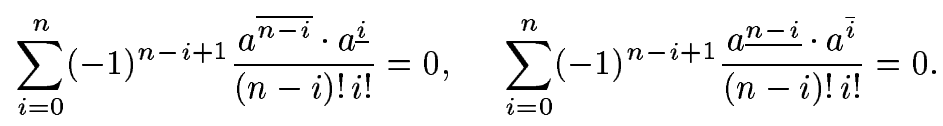

Следует отметить, что каждому $F$-параопределителю, значение которого известно, соответствуют некоторые комбинаторные тождества, которые можно получить, разлагая их по элементам первого столбца или по элементам последней строчки. При этом верхние и нижние $F$-параопределители генерируют двойственные тождества.

Приведем еще одну пару двойственных комбинаторных тождеств с возрастающими и убывающими факториальньми степенями:

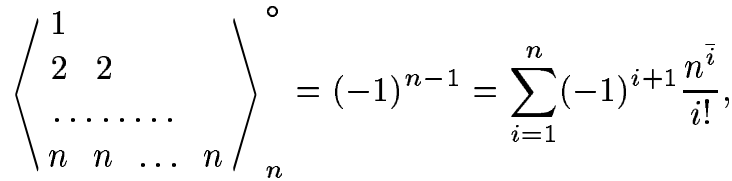

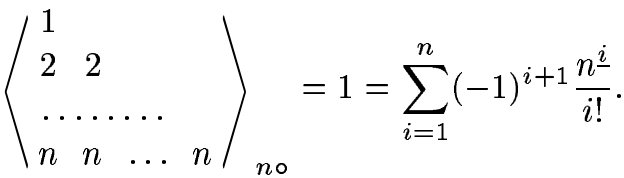

Пусть задан верхний $F$-параопределитель

$$
\diamond_{n}^{\circ}=\left\langle\begin{array}{lllllll}
2 & & & & & \\
1 & 2 & & & & \\
\ldots & \ldots & \ldots & & & \\
0 & 0 & \ldots & 1 & 2 & \\
0 & 0 & \ldots & 0 & 1 & 2
\end{array}\right\rangle_{n}
$$

Разложим его по элементам последней строчки, получим рекуррентную формулу

$$
\diamond_{n}^{\circ}=2 \cdot \diamond_{n-1}^{\circ}-\diamond_{n-2}^{\circ}
$$

с начальньми условиями $\diamond_{1}^{\circ}=2, \diamond_{2}^{\circ}=3$.

При помоши формулы $(5.15)$ легко найти значение верхнего $F$-параопределителя (5.14) и, следовательно, получить тождество

$$
\diamond_{n}^{\circ}=\sum_{i=0}^{[n / 2]}(-1)^{i} \frac{(i+1)^{\overline{n-2 i}}}{(n-2 i) !} \cdot 2^{n-2 i}=n+1
$$


6. Траектории на диаграммах Ферре и косых диаграммах. В этом пункте мы проиллюстрируем применение верхних $F$-параопределителей к нахождению числа кратчайших путей на диаграммах Ферре и косых диаграммах.

Пусть $\lambda=\left(\lambda_{1}, \lambda_{2}, \ldots, \lambda_{r}\right)$ - некоторое неупорядоченное разбиение натурального числа $n$. Разбиение $\mu=\left(\mu_{1}, \mu_{2}, \ldots, \mu_{r}\right)$ называется подразбиением разбиения $\lambda$ (обозначается $\mu \leqslant \lambda$ ), если выполняются неравенства $\mu_{i} \leqslant \lambda_{i}, i=1, \ldots, r$. Любой паре таких разбиений соответствует некоторая косая диаграмма (см. [3, с. 12-15])

$$
\operatorname{diagr}(\lambda, \mu)=\left(\begin{array}{l}
\lambda_{1}, \lambda_{2}, \ldots, \lambda_{s}, \ldots, \lambda_{r} \\
\mu_{1}, \mu_{2}, \ldots, \mu_{s}, 0, \ldots, 0
\end{array}\right)
$$

Если, например, $\lambda=(5,3,2,2), \mu=(2,1,1)$, то соответствующая диаграмма

$$
\operatorname{diagr}(\lambda, \mu)=\left(\begin{array}{l}
5,3,2,2 \\
2,1,1,0
\end{array}\right)
$$

имеет вид, указанньй на рис. 1.

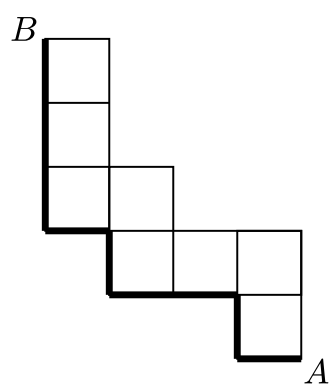

Рис. 1

Линию, которая ограничивает косую диаграмму снизу (сверху), назьвают ее нижними (верхними) ступеньками. На рис. 1 нижние ступеньки диаграммы выделены жирной линией. Под расстоянием между двумя точками диаграммы будем понимать кратчайшее расстояние между этими точками. Путем на косой диаграммемежду крайней юго-восточной точкой $A$ и крайней северо-западной точкой $B$ назовем кратчайший путь между этими точками. При этом будем двигаться только в двух направлениях $\uparrow$ и $\leftarrow$, т.е. будем рассматривать движения $\operatorname{mot}(\uparrow, \leftarrow)$.

Теорема 6.1. Число кратчайших траекторий мехду точками $A$ и $B$ на косой диаграмме

$$
\operatorname{diagr}(\lambda, \mu)=\left(\begin{array}{c}
\lambda_{1}, \ldots, \lambda_{n-1}, \lambda_{n} \\
\mu_{1}, \ldots, \mu_{n-1}, 0
\end{array}\right)
$$

равно

$$
L(\lambda / \mu)=L\left(\begin{array}{c}
\lambda_{1}, \ldots, \lambda_{n-1}, \lambda_{n} \\
\mu_{1}, \ldots, \mu_{n-1}, 0
\end{array}\right)=\operatorname{ddet}^{\circ}\left(a_{i j}\right)_{1 \leqslant j \leqslant i \leqslant n}
$$

əəe

$$
a_{i j}= \begin{cases}\lambda_{i}-\mu_{j}+j-i+1, & \text { если } \lambda_{i}-\mu_{j}+j-i+1 \geqslant 0 \\ 0, & \text { если } \lambda_{i}-\mu_{j}+j-i+1<0\end{cases}
$$




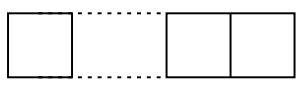

Рис. 2

ДокАЗАТЕльство. Очевидно, что число кратчайших путей на диаграмме на рис. 2 равно $k+1$, где $k$ - число клеток этой диаграммы.

Верхний $F$-параопределитель из теоремы 6.1, соответствующий этой диаграмме, имеет вид

$$
\left\langle\begin{array}{lllll}
2 & & & & \\
1 & 2 & & & \\
\ldots & \ldots & \ldots & & \\
0 & 0 & \ldots & 2 & \\
0 & 0 & \ldots & 1 & 2
\end{array}\right\rangle_{k}
$$

так как $\lambda_{1}=\cdots=\lambda_{k}=1 ; \mu_{1}=\cdots=\mu_{k}=0$. Поэтому его значение согласно (5.16) равно $k+1$.

Рассмотрим общую косую диаграмму

$$
\operatorname{diagr}(\lambda, \mu)=\left(\begin{array}{c}
\lambda_{1}, \ldots, \lambda_{n-1}, \lambda_{n} \\
\mu_{1}, \ldots, \mu_{n-1}, 0
\end{array}\right)
$$

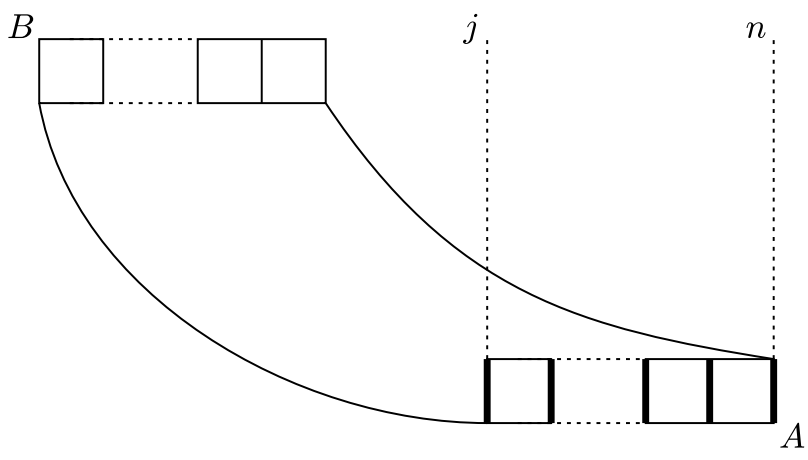

Рис. 3

На рис. 3 изображено $n-j+1$ клеток нижнего ряда этой диаграммы и $k$ клеток верхнего ее ряда. Очевидно, что число всех кратчайших путей от точки $A$ к точке $B$ на этой диаграмме равно сумме $n-j+2$ чисел $L(j-1), \ldots, L(n)$, которые соответственно равны числам кратчайших путей, проходящих через левую вертикальную сторону $j$-й клетки и каждую правую вертикальную сторону $i$-й, $i=j, \ldots, n$, клетки нижнего ряда диаграммы. На рис. 3 эти стороны выделены жирными линиями. Но число $L(i)$, $i=j-1, \ldots, n$, равно аналогичному числу кратчайших путей диаграммы, которая получается из диаграммы $\operatorname{diagr}(\lambda, \mu)$ в результате отбрасьвания всех клеток нижнего 
ряда и всех клеток этой диаграммы, лежащих правее $i$-го столбца. Следовательно, выполняется рекурсия

$$
\begin{gathered}
L\left(\begin{array}{l}
\lambda_{1}, \ldots, \lambda_{j-1}, \lambda_{j}, \lambda_{j+1}, \ldots, \lambda_{n} \\
\mu_{1}, \ldots, \mu_{j-1}, 0, \quad 0, \ldots, 0
\end{array}\right)=L\left(\begin{array}{c}
\lambda_{1}-1, \ldots, \lambda_{j-1}-1 \\
\mu_{1}-1, \ldots, \mu_{j-1}-1
\end{array}\right) \\
\quad+\sum_{i=j}^{n} L\left(\begin{array}{l}
\lambda_{1}-1, \ldots, \lambda_{j-1}-1, \lambda_{j}-1, \ldots, \lambda_{i}-1 \\
\mu_{1}-1, \ldots, \mu_{j-1}-1, \quad 0, \quad \ldots, 0
\end{array}\right)
\end{gathered}
$$

где

$$
\begin{aligned}
L(i) & =L\left(\begin{array}{l}
\lambda_{1}-1, \ldots, \lambda_{j-1}-1, \lambda_{j}-1, \ldots, \lambda_{i}-1 \\
\mu_{1}-1, \ldots, \mu_{j-1}-1, \quad 0, \ldots, 0
\end{array}\right), \\
L(j-1) & =L\left(\begin{array}{l}
\lambda_{1}-1, \ldots, \lambda_{j-1}-1 \\
\mu_{1}-1, \ldots, \mu_{j-1}-1
\end{array}\right), \quad i=j, \ldots, n .
\end{aligned}
$$

Таким образом, при помоши рекурсии (6.3) нахождение числа кратчайших путей после конечного числа шагов приводит к вычислению кратчайших путей на диаграммах вида рис. 2.

Докажем, что верхний $F$-параопределитель (6.1) при условии (6.2) удовлетворяет рекурсии (6.3). С этой целью для косых диаграмм

$$
\left(\begin{array}{c}
\lambda_{1}-1, \ldots, \lambda_{j-1}-1 \\
\mu_{1}-1, \ldots, \mu_{j-1}-1
\end{array}\right), \quad\left(\begin{array}{cc}
\lambda_{1}-1, \ldots, \lambda_{j-1}-1, \lambda_{j}-1, \ldots, \lambda_{i}-1 \\
\mu_{1}-1, \ldots, \mu_{j-1}-1, & 0,
\end{array}\right)
$$

из равенства (6.3) построим при помощи (6.2) соответственно матрицы

$$
\left.\begin{array}{c}
\left(\begin{array}{c}
\lambda_{1}-\mu_{1}+1 \\
\ldots \ldots \ldots \ldots \ldots \ldots \ldots \ldots \\
\lambda_{j-1}-\mu_{1}-j+3 \ldots
\end{array} \lambda_{j-1}-\mu_{j-1}+1\right.
\end{array}\right),
$$

Этим матрицам соответствуют верхние $F$-параопределители вида

$$
\operatorname{ddet}^{\circ}\left(R_{j-1,1}\right) \quad \text { и } \quad \operatorname{ddet}^{\circ}\left(R_{i 1}\left(\begin{array}{ccc}
j & \ldots & i \\
-1 & \ldots & -1
\end{array}\right)\right), j \leqslant i \leqslant n .
$$

Следовательно, нам необходимо доказать, что верхний $F$-параопределитель из теоремы 6.1 удовлетворяет равенству

$$
\operatorname{ddet}^{\circ}\left(a_{i j}\right)_{1 \leqslant j \leqslant i \leqslant n}=\operatorname{ddet}^{\circ}\left(R_{j-1,1}\right)+\sum_{i=j}^{n} \operatorname{ddet}^{\circ}\left(R_{i 1}\left(\begin{array}{ccc}
j & \ldots & i \\
-1 & \ldots & -1
\end{array}\right)\right)
$$


Прежде всего отметим, что $F$-параопределитель, в левой части равенства $(6.4)$, матрицу которого обозначим через $A$, является $F$-параопределителем смешанного типа, т.е. его часть с $j$-го по $n$-й столбец имеет вид верхнего $F$-параопределителя $\diamond 1_{n-j+1}^{\circ}$. Для лучшей наглядности отделим его вертикальной чертой. Следовательно, по утверждению 4.5 имеем равенство (4.11), из которого в силу того, что

$$
\operatorname{ddet}^{\circ}\left(R_{n, s+1}\right)-\operatorname{ddet}^{\circ}\left(R_{n ; s+1 \mapsto s}\right)=1, \quad s=j, \ldots, n-1,
$$

следует равенство

$$
\operatorname{ddet}^{\circ}(A)=\operatorname{ddet}^{\circ}\left(A\left(\begin{array}{c}
s \\
-1
\end{array}\right)\right)+\operatorname{ddet}^{\circ}\left(R_{s-1,1}\right),
$$

аналогичное равенству (5.4). Равенства (6.5) для $\operatorname{ddet}^{\circ}\left(R_{s-1,1}\right)$ при $s=j+1, \ldots, n$ имеют соответственно вид

$$
\begin{aligned}
& \operatorname{ddet}^{\circ}\left(R_{j 1}\right)=\operatorname{ddet}^{\circ}\left(R_{j 1}\left(\begin{array}{c}
j \\
-1
\end{array}\right)\right)+\operatorname{ddet}^{\circ}\left(R_{j-1,1}\right), \\
& \operatorname{ddet}^{\circ}\left(R_{j+1,1}\right)=\operatorname{ddet}^{\circ}\left(R_{j+1,1}\left(\begin{array}{c}
j+1 \\
-1
\end{array}\right)\right)+\operatorname{ddet}^{\circ}\left(R_{j, 1}\right), \\
& \operatorname{ddet}^{\circ}\left(R_{n 1}\right)=\operatorname{ddet}^{\circ}\left(R_{n 1}\left(\begin{array}{c}
n \\
-1
\end{array}\right)\right)+\operatorname{ddet}^{\circ}\left(R_{n-1,1}\right) .
\end{aligned}
$$

Складьвая их, получим равенство

$$
\operatorname{ddet}^{\circ}(A)=\sum_{i=j}^{n} \operatorname{ddet}^{\circ}\left(R_{i 1}\left(\begin{array}{c}
i \\
-1
\end{array}\right)\right)+\operatorname{ddet}^{\circ}\left(R_{j-1,1}\right)
$$

Но, так как

$$
\operatorname{ddet}^{\circ}\left(R_{n, s+1}\left(\begin{array}{c}
s+1 \\
-1
\end{array}\right)\right)-\operatorname{ddet}^{\circ}\left(R_{n ; s+1 \mapsto s}\right)=0 \quad \text { при } s=j, \ldots, n,
$$

то в силу равенства (4.11) получим равенство

$$
\operatorname{ddet}^{\circ}\left(R_{i 1}\left(\begin{array}{c}
i \\
-1
\end{array}\right)\right)=\operatorname{ddet}^{\circ}\left(R_{i 1}\left(\begin{array}{ccc}
j & \ldots & i \\
-1 & \ldots & -1
\end{array}\right)\right)
$$

Таким образом, равенство (6.6) примет вид равенства (6.4), что доказьвает теорему.

Так как вычеркивание клеток нижнего ряда косой диаграммы изменяет только элементы матрицы, лежащие по правую сторону от вертикальной черточки, которая разделяет матрицу смешанного вида, причем элементы угла $R_{n j}$ вследствие неравенств $\lambda_{i}>0,1 \leqslant j \leqslant i \leqslant n$, и равенства (6.2) положительны, то отрицательные элементы в матрице (6.1) можно заменить нулями. 


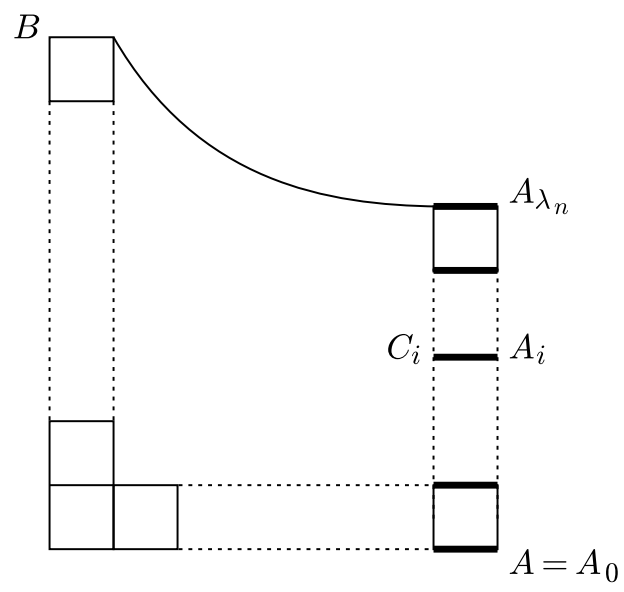

Рис. 4

ПримечАниЕ 6.1. Следует отметить тот очевидньй факт, что при симметрии диаграммы относительно биссектрисы первого квадранта, т.е. при переходе к сопряженной диаграмме (см. [3, с. 15]), число кратчайших путей не меняется. Поэтому вместо диаграммы, изображенной на рис. 2, можно рассматривать диаграмму, состоящую из $k$ клеток в одном столбце.

При этом имеем $\lambda_{1}=k, \mu_{1}=0$ и соответствующий ей $F$-параопределитель будет иметь вид $\langle k+1\rangle_{1}^{\circ}$. Следовательно, порядок $F$-параопределителя типа $\diamond 1$ иногда можно уменьшить, переходя к сопряженной диаграмме.

ПримечАниЕ 6.2. При $\mu_{1}=\cdots=\mu_{n-1}=0$ матрища смешанного типа переходит в матрицу типа $\diamond 1_{n}^{\circ}$, а косая диаграмма перейдет в диаграмму $е р p e$ (см. [4, с. 21], рис. 4).

ПримечАниЕ 6.3. При помощи диаграмм Ферре (рис. 4) можно получить новые интересные рекурсии для верхних $F$-параопределителей типа $\diamond 1_{n}^{\circ}$, а при помощи последних - новые комбинаторные тождества. Например, очевидно, что число всех кратчайших путей на диаграмме Ферре между точками $A$ и $B$ равно сумме всех кратчайших путей, проходящих через выделенные на рис. 4 отрезки $A_{i} C_{i}, i=0, \ldots, \lambda_{n}$. Следовательно, вьполняется рекурсия

$$
L\left(\begin{array}{ccc}
\lambda_{1}, & \ldots, & \lambda_{n} \\
0, & \ldots, & 0
\end{array}\right)=\sum_{i=0}^{\lambda_{n}} L\left(\begin{array}{ccc}
\lambda_{1}-i, & \ldots, & \lambda_{n-1}-i \\
0, & \ldots, & 0
\end{array}\right),
$$

которая в терминах верхнего $F$-параопределителя имеет вид

$$
\diamond 1_{n}^{\circ}=\sum_{i=0}^{\lambda_{n}} \operatorname{ddet}^{\circ}\left(R_{n-1,1}\left(\begin{array}{ccc}
1 & \ldots & n-1 \\
-i & \ldots & -i
\end{array}\right)\right) .
$$

Пусть, например, диаграмма Ферре имеет вид прямоугольника, т.е. $\lambda_{1}=\cdots=\lambda_{n}=k$; тогда верхний $F$-параопределитель, которьй равен числу кратчайших путей на этой диаграмме является $F$-параопределителем типа $\diamond 1$ и имеет вид

$$
\diamond 1_{n}^{\circ}=\left\langle\begin{array}{ccc}
k+1 & \\
k & k+1 & \\
\ldots \ldots \ldots \ldots \ldots \ldots \ldots \ldots & \ldots \ldots \ldots
\end{array}\right) .
$$


Применяя к $F$-параопределителю (6.7) утверждение 5.1 и учитьвая, что

$$
\operatorname{ddet}^{\circ}\left(R_{j 1}\left(\begin{array}{ccc}
1 & \ldots & j \\
-k & \ldots & -k
\end{array}\right)\right)=1 \quad \text { при } j=0, \ldots, n,
$$

получим равенство

$$
\diamond 1_{n}^{\circ}=\sum_{j=0}^{n} \frac{(j+1)^{\overline{k-1}}}{(k-1) !} \stackrel{(4.9)}{=} \frac{[n+1]^{k}}{k !}=\frac{(n+k) !}{n ! k !}
$$

7. Об одном частично упорядоченном множестве. В этом пункте устанавливается взаимно однозначное соответствие между подразбиениями $\mu$ разбиения $\lambda$ и кратчайшими путями на диаграмме $\operatorname{diagr}(\lambda, \mu)$. Таким образом, поскольку задача о кратчайших путях на косых диаграммах решена, решается задача установления мошности частично упорядоченного множества всех подразбиений некоторого разбиения.

Пусть $\lambda=\left(\lambda_{1}, \ldots, \lambda_{r}\right)$ - некоторое неупорядоченное разбиение натурального числа $n, \lambda_{1} \geqslant \lambda_{2} \geqslant \cdots \geqslant \lambda_{r}$. Множество $(R(\lambda), \leqslant)$ всех подразбиений разбиения $\lambda \mathrm{c}$ введенным на нем отношением порядка " $\leqslant$ " назовем частично упорядоченнылм множеством (ч.у.м.) подразбиений разбиения $\lambda$.

Элементы $\left(\mu_{1}, \ldots, \mu_{r}\right)$ одного уровня в диаграмме Хассе ч.у.м. $(R(\lambda), \leqslant)$ являются всеми возможными неупорядоченньми разбиениями натурального числа $m, 0 \leqslant m \leqslant n$, которые удовлетворяют условиям $\mu_{i} \leqslant \lambda_{i}, i=1, \ldots, r$.

ТЕОРема 7.1. Мощность ч.у.м. $(R(\lambda), \leqslant)$ равна числу всех кратчайших путей на әрафе Ферре разбиения $\lambda$.

ДокАЗАТЕЛЬСтво. Каждому подразбиению $\mu$ разбиения $\lambda$ соответствует единственньй кратчайший путь на графе Ферре разбиения $\lambda$, который совпадает с верхними ступеньками графа Ферре разбиения $\mu$, и наоборот. Теорема доказана.

Пусть $R(\mu, \lambda)$ - множество всех разбиений $\nu=\left(\nu_{1}, \nu_{2}, \ldots, \nu_{r}\right)$, компоненты которых удовлетворяют неравенствам

$$
\nu_{1} \geqslant \cdots \geqslant \nu_{r}, \quad \mu_{i} \leqslant \nu_{i} \leqslant \lambda_{i}, \quad i=1, \ldots, r
$$

Тогда, аналогично теореме 7.1, можно утверждать, что мощность множества $R(\mu, \lambda)$ равна числу кратчайших путей на диаграмме $\operatorname{diagr}(\lambda, \mu)$.

\section{СПИСОК ЦИТИРОВАННОЙ ЛИТЕРАТУРЫ}

[1] Заторський Р. А. Паравизначники та параперманенти трикутних матриць // Матем. студії. 2002. Т. 17. № 1. С. 45-54.

[2] Грэхем Р., Кнут Д., Паташник О. Конкретная математика. Основание информатики. М.: Мир, 1998.

[3] Макдональд И. Симметрические функции и многочлены Холла. М.: Мир, 1984.

[4] Эндрюс Г. Теория разбиений. М.: Наука, 1982. 